\title{
The Structure of Critical Care Transfer Networks
}

\author{
Theodore J. Iwashyna, MD, PhD, *† Jason D. Christie, MD, MSCE, $\$$ James Moody, PhD, 9 \\ Jeremy M. Kahn, MD, MSc, †ł and David A. Asch, MD, MBA †
}

\begin{abstract}
Rationale: Moving patients from low-performing hospitals to highperforming hospitals may improve patient outcomes. These transfers may be particularly important in critical care, where small relative improvements can yield substantial absolute changes in survival.
\end{abstract}

Objective: To characterize the existing critical care network in terms of the pattern of transfers.

Methods: In a retrospective cohort study, the nationwide 2005 Medicare fee-for-service claims were used to identify the interhospital transfer of critically ill patients, defined as instances where patients used critical care services in 2 temporally adjacent hospitalizations.

Measurements: We measured the characteristics of the interhospital transfer network and the extent to which intensive care unit patients are referred to each hospital in that network- - a continuous quantitative measure at the hospital-level known as centrality. We evaluated associations between hospital centrality and organizational, medical, surgical, and radiologic capabilities.

Results: There were 47,820 transfers of critically ill patients among 3308 hospitals. $4.5 \%$ of all critical care stays of any length involved an interhospital critical care transfer. Hospitals transferred out to a mean of 4.4 other hospitals. More central hospital positions were associated with multiple indicators of increased capability. Hospital characteristics explained $40.7 \%$ of the variance in hospitals' centrality.

Conclusions: Critical care transfers are common, and traverse an informal but structured network. The centrality of a hospital is associated with increased capability in delivery of services, suggesting that existing transfers generally direct patients toward better resourced hospitals. Studies of this network promise further improvements in patient outcomes and efficiency of care.

Key Words: networks, critical care, regionalization, Medicare

(Med Care 2009;47: 787-793)

From the *Division of Pulmonary and Critical Care, Department of Medicine, University of Michigan, Ann Arbor, Michigan; †Leonard Davis Institute of Health Economics, University of Pennsylvania, Philadelphia, Pennsylvania; †Division of Pulmonary, Allergy, and Critical Care, Department of Medicine, Hospital of the University of Pennsylvania, Philadelphia, Pennsylvania; §Center for Clinical Epidemiology and Biostatistics, University of Pennsylvania, Philadelphia, Pennsylvania; $\mid$ Department of Sociology, Duke University, Durham, North Carolina; and $\|$ Center for Health Equity Research and Promotion, Philadelphia VA Medical Center, Philadelphia, Pennsylvania.

Supported in part by NIH grants HL07891-09 and K08 HL09 1249 and an ATS Fellows Career Development Award.

Reprints: Theodore J. Iwashyna, MD, PhD, $3 \mathrm{a} 23300$ NIB, SPC 5419, 300 North Ingalls Building, Ann Arbor, MI 48109. E-mail: tiwashyn@umich.edu.

Copyright (C) 2009 by Lippincott Williams \& Wilkins

ISSN: 0025-7079/09/4707-0787
$\mathrm{C}^{\mathrm{n}}$ ritical illness is a common and growing part of American medicine. ${ }^{1} 22.4 \%$ of Americans use an intensive care unit (ICU) just before their death. ${ }^{2}$ A majority of patients with common conditions such as heart failure, malignancy, and hip fractures will use critical care. ${ }^{3}$ At the same time, there are wide variations between hospitals in the outcomes of critical care. $^{4-10}$ These variations in outcomes mean that in any given year thousands of patients who die in ICUs might have been saved, had they been in a different hospital. ${ }^{11,12}$

Such wide variation between hospitals has increased interest in moving patients to higher quality providers. Directing trauma victims to centers of excellence is associated with $25 \%$ to $50 \%$ improvements in outcomes. ${ }^{13,14}$ The Leapfrog Group recommends a similar approach for patients needing coronary artery bypass grafting and percutaneous coronary interventions. ${ }^{15}$ Others have called for a formal system to transfer patients with acute myocardial infarction ${ }^{16,17}$ or needing more general medical critical care. ${ }^{18}$

Whether or not formal systems of critical care transfer are implemented, patient transfers are already a common part of US critical care. Transfers between hospitals appear to be safe in appropriately staffed and equipped vehicles. ${ }^{19,20}$ However, we have almost no published information about the incidence or patterning of transfers of adult critically ill patients in the United States. Absent data on how often and where patients are being sent, it is difficult to plan quality improvement. For example, although some critically ill patients are moved from 1 hospital to another, are patients being systematically moved to hospitals that provide higher quality care? That is, does the informal transfer system work toward the same goals as formal regionalization?

In this article, we provide the first description of the informal system that has evolved for transferring critically ill Medicare beneficiaries among hospitals. Using nationwide data for 2005, we document the incidence of interhospital critical care transfers, and characterize the transfer network. In addition, we test 2 prespecified hypotheses about the flow of patients in the critical care network. "Centrality" is a hospital-level variable that quantifies the extent to which patients flow toward each hospital. ${ }^{21}$ Most fundamentally, hospitals transfer more patients to central hospitals. We define centrality formally under "Methods." We hypothesized that observable hospital organizational characteristics and capabilities (organizational, medical, surgical, and radiologic capabilities) are associated with hospital centrality. Second, we hypothesized that significant variation between hospitals' centrality in the network is associated with specified hospital characteristics. 


\section{METHODS}

\section{Analytic Approach}

We measured the interhospital transfer system using the tools of network analysis. Hospitals are the nodes in the network. Hospitals are connected by transfers of critically ill patients, which form pathways between nodes (formally termed "directed edges" in graph theory). ${ }^{22}$ These pathways can be 2 -way, if hospitals transfer back and forth with each other, or 1-way if 1 hospital transfers only out or only in. The pathways vary in their volume with the total number of patients moving in one direction or another between hospitals.

We used Medicare data to observe the network of critical care patient transfers and we used American Hospital Association (AHA) Annual Survey data to characterize the hospitals. We then measured the extent to which characteristics of the hospital were associated with that hospital's position in the critical care transfer network.

\section{Transfer Data: Medicare}

We used the final action claims from the 2005 Medicare Provider Analysis and Review file. Medicare claims and enrollment data capture $96 \%$ of the American population aged 65 and older. ${ }^{23}$ Critical care use is indicated directly in the hospital-filed claims. Although these data have some limitations, ${ }^{24}$ they have been used by one of $\mathrm{us}^{3}$ and others ${ }^{1}$ to measure critical care at both the patient and national levels. We excluded beneficiaries covered under certain types of group health organizations with capitated premiums who are not required to file claims. We excluded from consideration as critical care "psychiatric critical care" and intermediate or step-down units, ${ }^{24}$ but included medical, surgical, coronary care and burn units. We examined all claims for patients age 18 and above from the 50 United States and the District of Columbia, between September 1, 2004 and September 1, 2005.

\section{Hospital Data: AHA Annual Survey}

Since 1946, the AHA has conducted an annual census of all hospitals in the United States. ${ }^{25}$ This widely used electronic dataset contains self-reported data on hospital ownership, staffing, facilities, and capabilities. The survey's unit of analysis is the individual hospital, not the hospital or health care system with which a facility may be affiliated. The annual surveys achieve a high average response rate on each wave (83\% in 2001), with a higher rate among acute care hospitals. ${ }^{26}$ Where data were missing in 2005, we supplemented with the responses from 2003, giving data on $88.5 \%$ of the hospitals; this was possible for all variables except the presence of an interventional catheterization laboratory, for which we achieved complete data for $81.2 \%$ of hospitals.

The AHA data are extensively checked for internal consistency and consistency with past reports. With the exception of certain financial performance variables (particularly in for-profit hospitals), ${ }^{27}$ the data are considered highly reliable. ${ }^{28}$ Furthermore, to limit errors, we used only consistent and commonly used descriptions of facilities.

\section{Definition of Transfer}

Transfers between hospitals are not directly indicated in the claims. We defined a transfer as occurring between 2 hospitals A and $\mathrm{B}$ when a patient was observed to be in hospital A until a certain day, and then in hospital B beginning on the same day or the next day. Critical care transfers were defined as transfers that occurred between 2 hospitalizations, both of which involved critical care use. Distances between transfers are calculated using the Haversine formula, which takes into account the curvature of the earth. ${ }^{29}$

\section{Definition of a More Central Hospital}

The critical care transfer network is the aggregation of all interhospital transfers. A hospital's centrality in the network is the extent to which patients are transferred to it. ${ }^{30}$ A more central hospital is one that $(a)$ receives more patients from $(b)$ a greater number of hospitals that $(c)$ are themselves more central. An example is in Figure 1, and the formal recursive calculation is described in the Technical Appendix.

This measure of network centrality is analogous to the measure of web-page prominence PageRank used by the search engine Google. ${ }^{31,32}$ The search engine faces the problem of identifying the page for which the user is most likely looking; it assumes the user is looking for the most prominent webpage on a topic. A webpage to which many other webpages link is rated as more prominent. Likewise, a link coming from a webpage that is itself more prominent (eg, nytimes.com) is weighted more heavily than a link from a low visibility website (eg, the first author's little brother's home page). In the example in Figure 1, hospital $\mathrm{C}$ is more central than hospital $\mathrm{E}$, because $\mathrm{C}$ receives transfers from more hospitals than does $\mathrm{E}$. However, $\mathrm{F}$ is more central then $\mathrm{C}$, despite the fact that they both receive transfers from 2 other hospitals. This is because $\mathrm{C}$ transfers to $\mathrm{F}$, and $\mathrm{C}$ is itself more central than A or B.

\section{Statistical Analysis}

To summarize the extent to which hospital characteristics are associated with variation in network centrality, we used a nonparametric Kruskal-Wallis test to avoid unnecessary assumptions about the distribution of variables (Table 1). We used a $\chi^{2}$ test to evaluate the equivalence of the rates of characteristics across 4 hospital categories (Table 2). To test the relationship between the variation in network centrality and hospital characteristics, we used the $R^{2}$, functioning as a multiple correlation coefficient. Details on calculations and reports of sensitivity analyses are reported in the Technical Appendix.
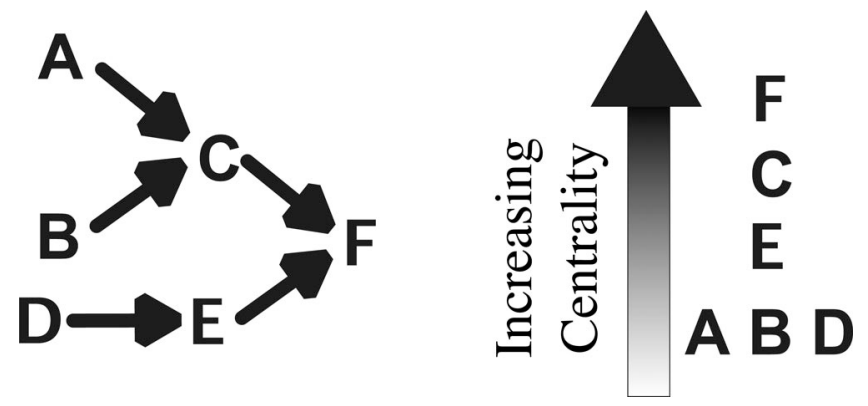

FIGURE 1. Example of calculation of network centrality. At left is a sample network, with all hospitals transferring the same number of patients. At right are the hospitals' relative centrality. 
TABLE 1. Characteristics of Hospitals and Association With Network Centrality

\begin{tabular}{|c|c|c|c|c|}
\hline & \multirow{2}{*}{$\begin{array}{l}\text { Percentage of Hospitals } \\
\text { With Characteristic }\end{array}$} & \multicolumn{2}{|c|}{$\begin{array}{l}\text { Kruskal-Wallis } \\
\text { Test }\end{array}$} & \multirow{2}{*}{$\begin{array}{l}\text { Relative Frequency Among } \\
\text { More Central Hospitals }\end{array}$} \\
\hline & & $x^{2}$ & $P$ & \\
\hline \multicolumn{5}{|l|}{ Organizational } \\
\hline Large hospital ( $>300$ beds) & $23.1 \%$ & $937.7^{*}$ & 0.0001 & + \\
\hline Small hospital ( $<100$ beds) & $52.5 \%$ & $485.8^{*}$ & 0.0001 & - \\
\hline For-profit & $18.9 \%$ & 6.3 & 0.0121 & - \\
\hline Residency program & $20.6 \%$ & 490.0 & 0.0001 & + \\
\hline Medical school affiliation & $31.4 \%$ & 433.9 & 0.0001 & + \\
\hline Medical/Surgical ICU & $92.2 \%$ & 52.9 & 0.0001 & + \\
\hline Coronary care & $47.0 \%$ & 649.7 & 0.0001 & + \\
\hline Associated Skilled Nursing Facility & $30.7 \%$ & 0.7 & 0.4114 & 0 \\
\hline \multicolumn{5}{|l|}{ Medical } \\
\hline Cardiac catheterization laboratory & $58.9 \%$ & 788.7 & 0.0001 & + \\
\hline Interventional cardiac laboratory & $46.9 \%$ & 947.2 & 0.0001 & + \\
\hline Hemodialysis & $43.3 \%$ & 440.7 & 0.0001 & + \\
\hline Neurology & $62.1 \%$ & 519.6 & 0.0001 & + \\
\hline Psychiatric emergency services & $44.3 \%$ & 229.4 & 0.0001 & + \\
\hline Chemotherapy delivered & $67.8 \%$ & 299.8 & 0.0001 & + \\
\hline Radiation therapy & $36.2 \%$ & 476.4 & 0.0001 & + \\
\hline \multicolumn{5}{|l|}{ Surgical } \\
\hline Trauma center (level 1 or 2 ) & $21.1 \%$ & 430.9 & 0.0001 & + \\
\hline Adult cardiac surgery & $36.3 \%$ & 1157.6 & 0.0001 & + \\
\hline Transplant & $8.9 \%$ & 418.3 & 0.0001 & + \\
\hline Orthopedics & $84.5 \%$ & 113.0 & 0.0001 & + \\
\hline \multicolumn{5}{|l|}{ Diagnostic radiology } \\
\hline Computed tomography scanner & $95.6 \%$ & 9.6 & 0.0019 & + \\
\hline Magnetic resonance imaging & $79.3 \%$ & 103.3 & 0.0001 & + \\
\hline Diagnostic radioisotope & $81.6 \%$ & 129.2 & 0.0001 & + \\
\hline
\end{tabular}

*Compares large hospitals to all others and small hospitals to all others; equivalent results were obtained for ordinal variable small/medium/large.

TABLE 2. Characteristics of Hospitals that Send and Receive Transfers

\begin{tabular}{lrccccc}
\hline & Sender & Receiver & Both & No Transfers & $\begin{array}{c}\text { Test for } \\
\text { Equivalence Cross } \\
\text { All 4 Groups }\end{array}$ \\
\hline $\mathrm{n}$ & 907 & 30 & 2371 & 557 & $\frac{\chi^{2}}{223.3}$ & $\frac{P}{<0.001}$ \\
Large hospital ( $>300$ beds) & $2.8 \%$ & $20.0 \%$ & $32.9 \%$ & $23.7 \%$ & 384.4 & $<0.001$ \\
Small hospital ( $<100$ beds) & $55.2 \%$ & $13.3 \%$ & $15.5 \%$ & $25.3 \%$ & 132.3 & $<0.001$ \\
Residency program & $5.5 \%$ & $60.0 \%$ & $26.1 \%$ & $19.6 \%$ & 150.9 & $<0.001$ \\
Medical school affiliation & $14.2 \%$ & $66.7 \%$ & $39.1 \%$ & $26.0 \%$ & 326.8 & $<0.001$ \\
Adult cardiac surgery & $4.7 \%$ & $90.0 \%$ & $45.2 \%$ & $39.7 \%$ & &
\end{tabular}

\section{RESULTS}

Three thousand four hundred thirty-nine hospitals cared for 2,138,416 critical care hospitalizations in the United States in fee-for-service Medicare during 2005. There were 47,820 transfers of critically ill patients among 3308 hospitals. That is, nearly all hospitals participated in at least 1 transfer, and $4.5 \%$ of all critical care hospitalizations involved such a transfer. The characteristics of the hospitals involved in the transfer networks are described in Table 1 . Transfers tended to occur relatively early during the stay in the first hospitalization. Median length of stay at the sending hospital was 2 days (mean: $4.6 \pm 7.2$ days standard deviation). Median length of stay at the receiving hospital was 8 days (mean: $11.5 \pm 12.9$ days standard deviation).

Three thousand three hundred six hospitals transferred among themselves in a single interconnected component; 2 hospitals transferred reciprocally to each other but not to the main component. $27.4 \%$ (907) sent but did not receive critical care transfers. $71.7 \%$ (2371) received and sent critical care transfers. $0.9 \%$ (30) only received critical care transfers. Only $0.2 \%$ of hospital pairs transfer 
Network Neighborhood of Most Central Dallas ICU

FIGURE 2. Map of transfer network. Hospitals are shown at their latitude and longitude in a simple Cartesian projection. Node size is proportional to centrality. Line darkness is proportional to the number of transfers passing between the 2 hospitals.

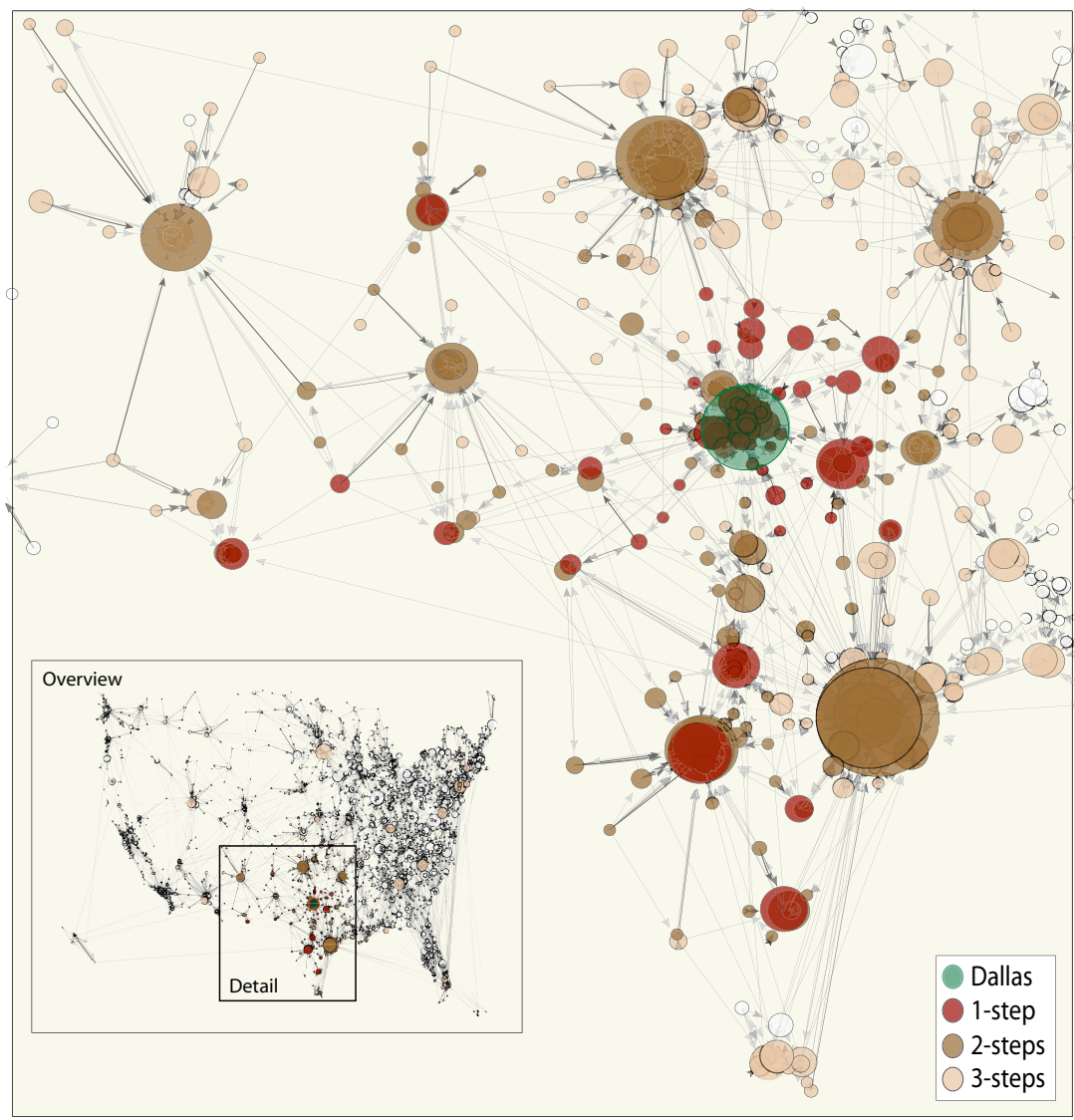

patients back and forth between each other; most transfers flow in 1 direction. Selected characteristics of the hospitals are shown in Table 2. Hospitals that only send were smaller, less likely to have a residency program or medical school affiliation, and less likely to possess advanced surgical capacity. Hospitals that only receive were larger and had more of these capacities. Hospitals that both sent and received tend to be in the middle of these 2 extremes.

The US network is represented graphically in Figure 2. The substantial interconnections between metropolitan areas - indeed between regions - is evident both in the national map and in the area of detail, chosen to give greater perspective on the variety of transfer relationships between hospitals. The median patient was transferred 29.2 miles, with an interquartile range of 11.6 to 54.3 miles. The mean transfer distance was 45.7 miles (Table 3 ).

TABLE 3. Distance of Interhospital Transfer

\begin{tabular}{lc}
\hline Distance (miles) & Percentage of Transfers \\
\hline $0-5$ & $10.2 \%$ \\
$5-10$ & $11.7 \%$ \\
$10-25$ & $22.3 \%$ \\
$25-50$ & $27.3 \%$ \\
$50-100$ & $19.9 \%$ \\
$>100$ & $8.6 \%$ \\
\hline
\end{tabular}

Hospitals vari ${ }^{1}$ ed significantly in the number of other hospitals to which they sent and from which they received critically ill patients, as shown in Figure 3. As shown in part A, 721 hospitals received transfers from only 1 other hospital; 5 hospitals received transfers from more than 75 other hospitals during 2005. As shown in part B, 30 hospitals transferred no patients out, 261 hospitals transferred to only 1 other hospital, and at the other extreme, 111 hospitals transferred to 10 or more other hospitals. The median hospital sent 11 total Medicare patients out to 4 other hospitals (mean: 4.4 hospitals \pm 2.5 standard deviation).

Hospital characteristics and capabilities were strongly associated with centrality in the transfer network. As shown in Table 1, network centrality was associated with nearly all prespecified organizational, medical, surgical, and radiologic services. More central hospitals were more capable in each of these bivariate associations. The presence of a skilled nursing facility (SNF) at the hospital - which was hypothesized not to be associated with centrality (eg, a negative control) — was not associated with centrality. The 26 hospital variables accounted for $40.7 \%$ of the variation between hospitals in their centrality.

\section{DISCUSSION}

This study has several main findings. First, interhospital transfers of critically ill patients are common in fee-for- 


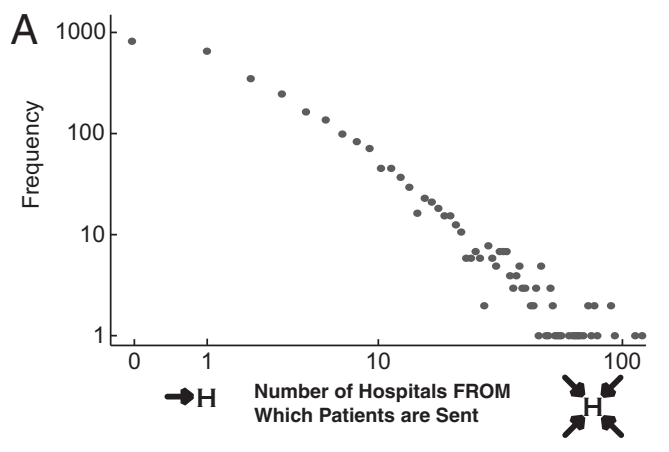

\begin{tabular}{|c|c|c|}
\hline$\underline{\text { In-Degree }}$ & Frequency & Percentage \\
\hline 0 & 907 & $27.4 \%$ \\
\hline 1 & 721 & $21.8 \%$ \\
\hline 2 & 382 & $11.6 \%$ \\
\hline $3-5$ & 445 & $13.5 \%$ \\
\hline $5-10$ & 514 & $15.5 \%$ \\
\hline $11-50$ & 319 & $9.6 \%$ \\
\hline $51-75$ & 15 & $0.5 \%$ \\
\hline$>75$ & 5 & $0.2 \%$ \\
\hline
\end{tabular}

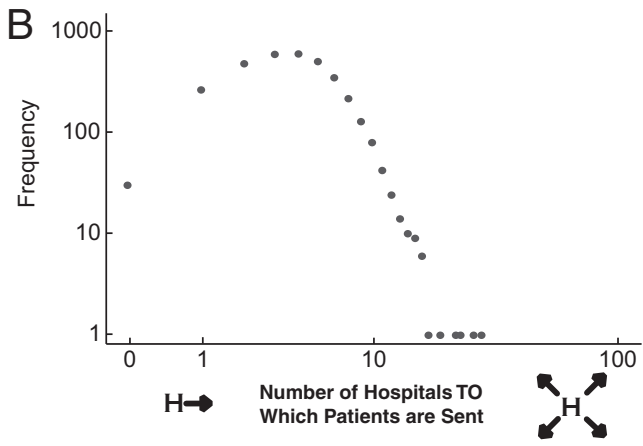

FIGURE 3. Patterns of connections. Panel A, displays the number of other hospitals that transferred patients into each hospital, formally termed "in-degree." Equivalent data is presented in tabular form to the right. Both horizontal and vertical axes are logarithmic due to wide variation. Panel $\mathbf{B}$, shows the number of other hospitals to which each hospital transferred patients out, formally termed "out-degree." service Medicare, involving approximately 1 in 20 Medicare critical care stays of any length. Second, hospitals transfer critically ill patients to several other hospitals, in contrast to the hub-and-spoke model often invoked to conceptually explain secondary and tertiary care. Third, the network appears to systematically move patients toward better resourced hospitals.

These findings reveal the existence of an organic network of critical care transfers. Our main interpretation is that network analysis of the type reported in this paper can help define the criteria for a successful system of critical care transfers as well as suggest efficient ways to get there. For example, we observe that more central hospitals have more technologic capability, suggesting that the general flow of critically ill patients is toward better resourced hospitals. However, not all patients flow in this direction. Some move in the opposite direction and many patients do not move at all. Whether we rely on measures of hospital resources or, in the future, measures of hospitals' clinical performance, the ICU transfer network we observe among Medicare beneficiaries reveals substantial promise for quality improvement. We might ask which patients should remain where they are, which should be transferred to better performing hospitals, and which, perhaps, should be sent to less resourced hospitals to improve efficiency or free capacity for sicker patients who need it more.

Approximately 1 in 1000 of possible interhospital connections actually occurred during 2005. In a low-density network such as this, careful analysis may reveal qualityimproving transfer relationships that are not currently established. Some hospitals may not be transferring patients to nearby facilities able to provide better care; some highly resourced hospitals may not be accepting patients from nearby less-capable hospitals. Some hospitals may not cur- rently have optimal capabilities to support the load of incoming critically ill patients, and one could target those hospitals for improvement or redirection of patients. No current incentive arrangement suggests that the existing network will optimize itself, but network analyses can help policy makers see and shape an aggregate structure that is invisible to the individual hospitals and patients. Network analyses are widely used in other areas, including sociology, ${ }^{22}$ physics, ${ }^{33}$ molecular biology, ${ }^{33}$ management, ${ }^{34}$ and military operations, ${ }^{35,36}$ but only rarely in the study of health care systems. ${ }^{37-40}$

The existing transfer system is not a simple hierarchy. Small community hospitals do not exist as satellites of single local centers, which then refer to dominant regional centers. This is true even within fee-for-service Medicare, so the heterogeneity of referrals is not the result of differing insurance plans within a hospital. While the secondary/tertiary hospital model may have some heuristic value, the implied hierarchy (in which secondary hospitals send but do not receive patients and tertiary hospitals receive but do not send patients) does not appear to be present in our data. Instead, hospitals appear to maintain diverse portfolios of other facilities to which they transfer patients. There are several potential reasons for this. There may be significant congestion at referral centers, with more central hospitals not able to accept all patients in a timely manner. Providers may be optimizing their referral patterns for local expertise based on particular patients' illness. Families may have important roles in choosing closer, more prestigious, or better advertised hospitals. Differentiating among these hypotheses is essential to evaluating the extent to which the system is working to meet diverse, legitimate values, or is simply operating inefficiently. 
The present work has a number of limitations. First, our data are derived from Medicare for a single year, and thus we cannot evaluate whether transfer patterns are different for non-Medicare patients or how they might change over time. However, over half of ICU patients are elderly, ${ }^{41}$ and Medicare is a domain of public policy interest in its own right. Second, our data reflect transfers between critical care hospitalizations; limitations in the data prevent us from examining only direct ICU-to-ICU transfers. It is possible that some of the transfers we have identified include patients who were discharge from the ICU to the floor at the first hospital, then transferred to a second hospital floor bed where they decompensated, requiring a distinct episode of ICU care. Our data cannot estimate the frequency with which this occurs. As a sensitivity test, we repeated our analyses using only transfers where the patients were in the initial sending hospital for 1 day, and the patterns we report were all replicated. Third, while we have demonstrated that patients are in general transferred toward better-resourced hospitals, further work remains to estimate the effects of transfer on quality and cost using patient-level data, and how those outcomes might be further improved. Fourth, interhospital transfers are one part of the system for transferring critically ill patients, which includes transfers directly from emergency departments and, indeed, field triage by EMS units. A comprehensive approach to improving the efficiency of care for the critically ill will complement the analyses presented here with similarly detailed examinations of other areas and then integrate across systems.

Network analyses such as these provide structure, metrics, and analytic tools to characterize and improve the relationships among hospitals. These networks likely have implications for the efficiency and adaptability of providers, ${ }^{42}$ the diffusion of innovation, ${ }^{43}$ and the spread of infections and other conditions. ${ }^{44}$ As we work toward a health care system that provides the best patient care with existing resources, the network analysis in this study reveals how much we have achieved already, and how much more we might achieve as well.

\section{ACKNOWLEDGMENTS}

The authors thank Maxim Herlim for providing expert programming.

\section{REFERENCES}

1. Halpern NA, Pastores SM, Greenstein RJ. Critical care medicine in the United States 1985-2000: an analysis of bed numbers, use, and costs. Crit Care Med. 2004:32:1254-1259.

2. Angus DC, Barnato AE, Linde-Zwirble WT, et al. Use of intensive care at the end of life in the united states: an epidemiologic study. Crit Care Med. 2004;32:638-643.

3. Iwashyna TJ. Critical care use during the course of serious illness. Am J Respir Crit Care Med. 2004;170:981-986.

4. Chen EW, Canto JG, Parsons LS, et al. Relation between hospital intra-aortic balloon counterpulsation volume and mortality in acute myocardial infarction complicated by cardiogenic shock. Circulation. 2003;108:951-957.

5. Kahn JM, Goss CH, Heagerty PJ, et al. Hospital volume and the outcomes of mechanical ventilation. $N$ Engl J Med. 2006;355:41-50.

6. Canto JG, Every NR, Magid DJ, et al. The volume of primary angioplasty procedures and survival after acute myocardial infarction. $N$ Engl $J$ Med. 2000;342:1573-1580.

7. Magid DJ, Calonge BN, Rumsfeld JS, et al. Relation between hospital primary angioplasty volume and mortality for patients with acute mi treated with primary angioplasty vs thrombolytic therapy. JAMA. 2000; 284:3131-3138.

8. McGrath PD, Wennberg DE, Dickens JD Jr, et al. Relation between operator and hospital volume and outcomes following percutaneous coronary interventions in the era of the coronary stent. JAMA. 2000; 284:3139-3144.

9. Hannan EL, Wu C, Walford G, et al. Volume-outcome relationships for percutaneous coronary interventions in the stent era. Circulation. 2005; 112:1171-1179.

10. Nathens AB, Jurkovich GJ, Maier RV, et al. Relationship between trauma center volume and outcomes. JAMA. 2001;285:1164-1171.

11. Krumholz HM, Normand S-LT, Spertus JA, et al. Measuring performance for treating heart attacks and heart failure: the case for outcomes measurement. Health Aff. 2007;26:75-85.

12. Kahn JM, Linde-Zwirble WT, Wunsch $H$, et al. Potential value of regionalized intensive care for mechanically ventilated medical patients. Am J Respir Crit Care Med. 2008;177:285-291.

13. Durham R, Pracht E, Orban B, et al. Evaluation of a mature trauma system. Ann Surg. 2006;243:775-785.

14. MacKenzie EJ, Rivara FP, Jurkovich GJ, et al. A national evaluation of the effect of trauma-center care on mortality. N Engl J Med. 2006;354: 366-378.

15. Birkmeyer JD, Dimick JB. Leapfrog Safety Standards: Potential Benefits of Universal Adoption. Washington, DC: The Leapfrog Group; 2004.

16. Hannan EL. Evaluating and improving the quality of care for acute myocardial infarction can regionalization help? JAMA. 2006;295:2177-2179.

17. Jollis JG, Roettig ML, Aluko AO, et al. Implementation of a statewide system for coronary reperfusion for st-segment elevation myocardial infarction. JAMA. 2007;298:220. joc70124.

18. Barnato AE, Kahn JM, Rubenfeld GD, et al. Prioritizing the organization and management of intensive care services in the United States: the PrOMIS Conference. Crit Care Med. 2007;35:1003-1006.

19. American College of Emergency Physicians. Interfacility transportation of the critical care patient and its medical direction. Ann Emerg Med. 2006;47:305.

20. Fan E, McDonald RD, Adhikari NKJ, et al. Outcomes of interfacility critical care adult patient transport: a systematic review. Crit Care. 2006;10:R6.

21. Freeman LC. Centrality in social networks: conceptual clarification. Soc Network. 1978/1979;1:215-239.

22. Wasserman S, Faust K. Social Network Analysis: Methods and Applications. Cambridge: Cambridge University; 1994.

23. Hatten J. Medicare's common denominator: the covered population. Health Care Financ Rev. 1980;2:Fall 53-64.

24. Halpern NA, Pastores SM, Thaler HT, et al. Critical care medicine use and cost among Medicare beneficiaries 1995-2000: major discrepancies between two United States federal Medicare databases. Crit Care Med. 2007;35:692-699.

25. Kralovec PD, mullner R. The American Hospital Association's Annual Survey of Hospitals: continuity and change. Health Serv Res. 1981;16: 351-355.

26. American Hospital Association. American Hospital Association Annual Survey Database for Fiscal Year 2001. Chicago, IL: American Hospital Association; 2001.

27. Mitchell S, Spetz J, Seago JA. Errors in data on hospital ownership. Inquiry. 2001/2002;38:432-439.

28. Mullner R, Chung K. The American Hospital Association's Annual Survey of Hospitals: a critical appraisal. J Consum Mark. 2002;19:614618.

29. Sinnott RW. Virtues of the Haversine. Sky Telescope. 1984;68:159. Available at: http://www.movable-type.co.uk/scripts/gis-faq-155.151.html.

30. Knoke D, Burt RS. Prominence. In: Burt RS, Minor MJ, eds. Applied Network Analysis: A Methodological Introduction. Beverly Hills, CA: Sage. 1983;195-222.

31. Brin S, Page L. The anatomy of a large-scale hypertextual web search engine. WWW7/Comput Networks. 1998;30:110-117.

32. Page L, Brin S, Motwani R, et al. The pagerank citation ranking: bringing order to the web. 1999. Available at: http://dbpubs.stanford. edu/pub/1999-66.

33. Newman MEJ, Barabasi A-L, Watts DJ. The Structure and Dynamics of Networks. Princeton, NJ: Princeton University Press; 2006. 
34. Cross RL, Parker A. The Hidden Power of Social Networks: Understanding How Work Really Gets Done in Organizations. Cambridge, MA: Harvard Business School Press; 2004.

35. Petraus DH, Amos JF. The US Army/Marine Corps Counterinsurgency Field Manual. Chicago, IL: University of Chicago Press; 2007.

36. Cebrowski AK, Garstka JJ. Network-centric warfare: its origin and future. US Naval Inst Proc. 1998;124:28-35.

37. Keating NL, Ayanian JZ, Cleary PD, et al. Factors affecting influential discussions among physicians: a social network analysis of a primary care practice. J Gen Intern Med. 2007;22:794-798.

38. Gibbons DE. Interorganizational network structures and diffusion of information through a health system. Am J Public Health. 2007;97: $1684-1692$.

39. Powell WW, Koput KW, Smith-Doerr L. Interorganizational collaboration and the locus of innovation: networks of learning in biotechnology. Adm Sci Q. 1996;41:116-145.

40. Westphal JD, Gulati R, Shortell SM. Customization or conformity? an institutional and network perspective on the content and consequences of tqm adoption. Adm Sci Q. 1997;42:366-394.

41. Angus DC, Shorr AF, White A, et al. Critical care delivery in the United States: distribution of services and compliance with Leapfrog recommendations. Crit Care Med. 2006;34:1016-1024.

42. Burt RS. Structural holes and good ideas. Am J Sociol. 2004;110:349399.

43. Rogers EM. Diffusion of Innovations. New York, NY: Free Press; 2003.

44. Christakis NA, Fowler JH. The spread of obesity in a large social network over 32 years. $N$ Engl J Med. 2007;357:370-379.

45. Bonacich P. Power and centrality: a family of measures. Am J Sociol. 1987;92:1170-1182.

46. Moody J. SPAN: SAS Programs for Analyzing Networks. Durham, NC: Duke University; 2000.

47. Batagelj V, Mrvar A. Pajek: Program for Analysis and Visualization of Large Networks. Ljubljana, Slovenia: University of Ljubljana; 2006.

48. de Nooy W, Mrvar A, Batagelj V. Exploratory Social Network Analysis With Pajek. Cambridge: Cambridge University Press; 2005.

49. StataCorp. Stata Statistical Software: Release 8. College Station, TX Stata Corporation; 2006.

\section{APPENDIX}

\section{Calculation of Centrality Scores}

For the calculation of centrality, we use the widely adopted score of Bonacich. ${ }^{45}$ The transfer network is represented by an asymmetric square matrix, referred to as the relationship matrix $R$. In our case, this is a 3306 by 3306 matrix for the main component of the network. The element $R_{i, j}$ is the number of patients transferred from hospital $i$ to hospital $j . R_{i, j}$ equals 0 if no transfers occurred in that direction. The centrality of all hospitals are calculated as a vector, in matrix notation.

$$
\text { Centrality }=\alpha(I-\beta R)^{-1} R 1
$$

where $\alpha$ is an arbitrary scaling parameter, $I$ is the identity matrix, $\beta$ is a dependence parameter, and 1 is a vector of $1 \mathrm{~s}$. The dependence parameter indicates the extent to which a hospital's centrality increases with increasing centrality of those hospitals that refer to it. With higher dependence parameters, the centrality of referring hospitals is given more weight in the calculation. This is equivalent to a recursive definition at the level of individual hospitals.

$$
\text { Hospital } j \text { 's centrality }=\mathrm{c}_{j}=\sum\left(\alpha+\beta \mathrm{c}_{i}\right) R_{i j} \text { over all } i \neq j
$$

where $c_{i}$ is the centrality of each other hospital $i$ and $R_{i j}$ is the number of patients transferred from hospital $i$ to hospital $j$.

We conducted our primarily analyses with a dependence parameter of 0.025 , the largest value for which the parameter does not exceed the inverse of the largest eigenvalue of the input matrix, as is typically done. We performed sensitivity tests of our results for a range of plausible Bonacich centrality measures, with dependence parameters of 0 and 0.01 . Hospitals not in the main component ( 2 of 3308) were excluded from the centrality analyses, as the values do not converge in a disconnected matrix.

\section{Statistical Analysis, Including Sensitivity Analyses}

To test the association between hospital characteristics and centrality, we used a nonparametric Kruskal-Wallis test to avoid unnecessary assumptions about the distribution of variables. We tested the robustness of our results to assumptions about the appropriate Bonacich centrality score and assumptions about missing data. For-profit status was marginally associated with centrality $(P=0.0121)$, and this association was inconsistent across alternative centrality measures. All other results were robust to alternative centrality measures. The lack of association between a SNF and centrality was sensitive to extreme assumptions about missing data, that is, the association became significant if all of the hospitals missing data were assumed to have a SNF, but not if all were assumed not to have a SNF. All other results were similar across missing data assumptions.

To test the relationship between the variation in network centrality and hospital characteristics, we used the $R^{2}$, functioning as a multiple correlation coefficient. Because the various hospital characteristics do not represent meaningful independent counterfactuals (eg, the effect of being a transplant center independent of having a computed tomography scanner) and were not germane to our prespecified hypotheses, we did not present the coefficients of multiple regression.

Data extraction and computation of network centrality were performed in SAS using SPAN for SAS. ${ }^{46}$ Network visualization was done using Pajek. ${ }^{47,48}$ Statistical analyses were conducted in Stata version 9.2. ${ }^{49}$ 